\title{
More is less, less is more, or does it really matter? The curious case of impact of azacitidine administration schedules on outcomes in patients with myelodysplastic syndromes
}

\author{
Rory M. Shallis ${ }^{1,3}$ and Amer M. Zeidan ${ }^{1,2,3^{*}}$ (i)
}

\begin{abstract}
Myelodysplastic syndromes (MDS) encompass a diverse group of hematologic disorders characterized by ineffective and malignant hematopoiesis, peripheral cytopenias and significantly increased risk of progression to acute myeloid leukemia (AML). The hypomethylating agents (HMA) azacitidine and decitabine induce meaningful clinical responses in a significant subset of patients with MDS. Though never compared directly with decitabine, only azacitidine has improved overall survival (OS) compared to conventional care in a randomized trial in patients with higher-risk MDS. The azacitidine regimen used in this pivotal trial AZA-001 included administration at $75 \mathrm{mg} / \mathrm{m}^{2} /$ day for 7 consecutive days in 28-day cycles (7-0 regimen). Given the logistical difficulties of weekend administration in the 7-0 regimen, as well as in efforts to improve response rates, alternative dosing schedules have been used. In a typical 28-day cycle, administration schedules of 3, 5, 10, and (with the oral version of azacitidine) 14 and 21 days have been used in clinical trials. Most trials that evaluated alternative administration schedules of azacitidine did so in lower-risk MDS and did not directly compare to the 7-0 schedule. Given the lack of randomized prospective studies comparing the 7-0 schedule to the other regimens of azacitidine in MDS, Shapiro et al. conducted a systematic review in an attempt to answer this question. Here we place the findings of this important work in clinical context and review the current knowledge and unresolved issues regarding the impact of administration schedules of azacitidine on outcomes of patients with both lower-risk and higher-risk MDS.
\end{abstract}

Keywords: Myelodysplastic syndrome, MDS, Azacitidine, Decitabine, Hypomethylating agents, HMAs

\section{Background}

Myelodysplastic syndromes (MDS) comprise a heterogenous subset of the myeloid malignancies and are characterized by disordered and malfunctional hematopoiesis, subsequently leading to clinically significant cytopenias and a substantial risk of progression to acute myeloid leukemia (AML) [1]. Treatment with the hypomethylating agents (HMAs) azacitidine (5-azacytidine) and decitabine (5-aza-2'-deoxycytidine)

\footnotetext{
* Correspondence: amer.zeidan@yale.edu

${ }^{1}$ Department of Internal Medicine, School of Medicine, Yale University, New Haven, CT, USA

${ }^{2}$ Cancer Outcomes, Public Policy, and Effectiveness Research (COPPER)

Center, Yale University, New Haven, CT, USA

Full list of author information is available at the end of the article
}

leads to objective clinical responses in nearly half of patients with MDS, and delays progression to AML [2-6]. The two HMAs have never been prospectively compared head-tohead with regards to overall response rate (ORR; the sum of complete response $[\mathrm{CR}]$, partial response $[\mathrm{PR}]$, and hematological improvement $[\mathrm{HI}]$ according to the International Working Group (IWG) 2006 criteria) or their effect on survival [7].

\section{Main text}

The early phase cancer and leukemia group B [CALGB] trials demonstrated favorable responses to azacitidine in about half the patients [Table 1]; many of them had excess blasts $[2,3]$. The CALGB9221 trial was the first 


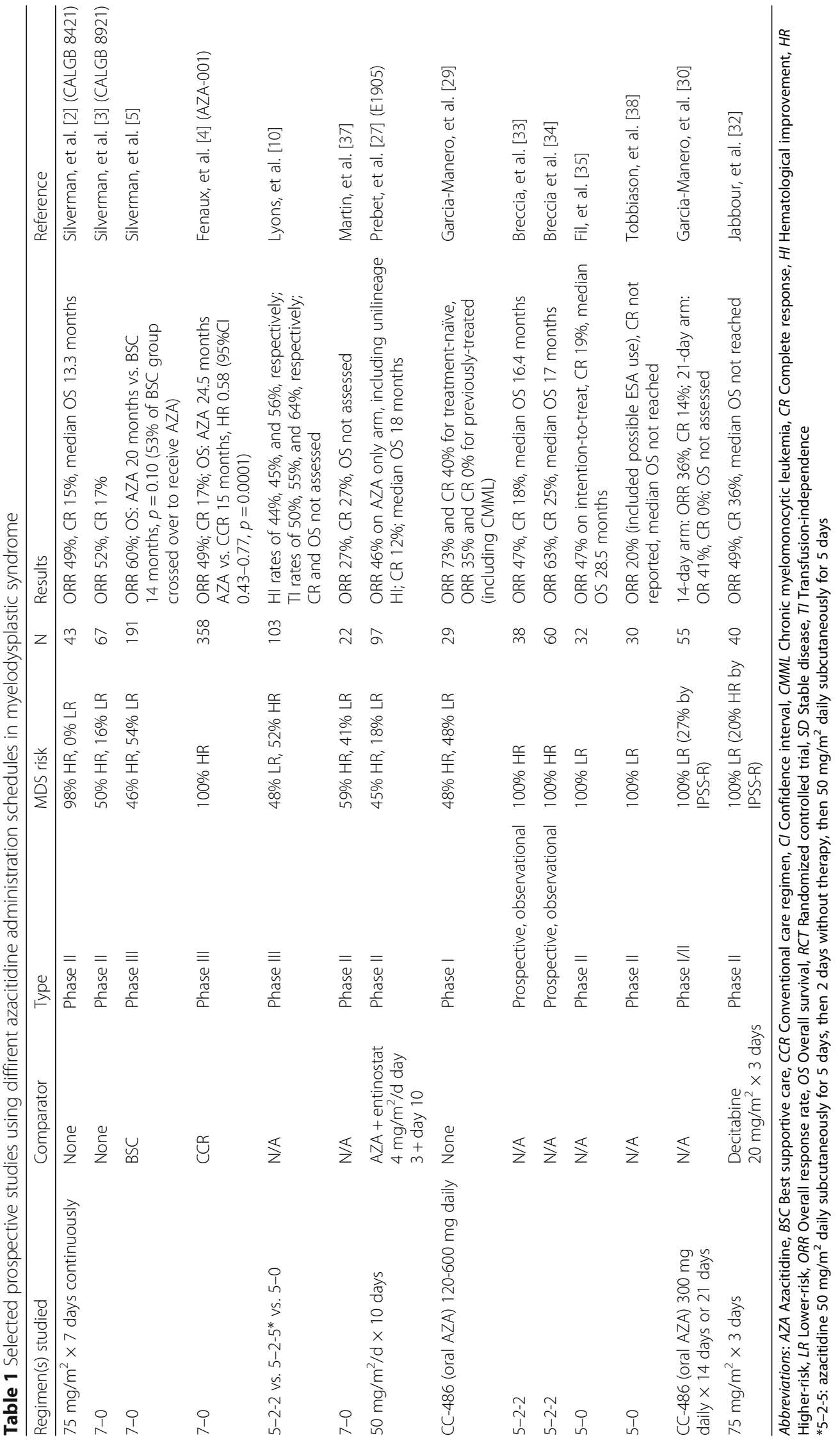


randomized phase III trial of azacitidine in MDS, and showed that azacitidine had an ORR of $60 \%$ and delayed progression to AML compared to best supportive care [BSC] [5]. Although there was no impact on overall survival, potentially because the trial allowed early crossover to azacitidine, the drug was approved for treatment of MDS in the United States in 2004. The landmark AZA-001, published in 2009, was the first and only randomized phase III trial to demonstrate an OS advantage with a drug (azacitidine, $N=179$ ) compared to conventional care regimens ( $C R R, N=179$, which included pre-selected BSC [59\%], low-dose cytarabine [27\%], or intensive chemotherapy [14\%]) in patients with international prognostic scoring system (IPSS) intermediate-2 or high risk MDS (24.5 months vs. 15.0 months; hazard ration $=0.58, p=0.0001$ ) [4]. The survival advantage was confirmed in each subset of response, including those whose best response was HI [8]. Based on the results of this seminal study, the European Medicines Agency approved azacitidine for use in higher-risk MDS (i.e. IPSS intermediate- 2 and high risk). Both phase 3 studies used the standard schedule of 7 consecutive days of azacitidine at $75 / \mathrm{m}^{2} /$ day in 28 -day cycles (the so called $7-0$ regimen). While decitabine is also approved in USA for use in MDS based on improvement in response rates and delayed progression to AML, neither of the two large randomized trials that compared decitabine to BSC in MDS showed a significant difference in OS $[6,9]$.

The approved regimen of azacitidine (7-0) requires weekend administration and poses logistical challenges to both infusion centers and patients. The obligation to arrange weekend administration is difficult for community practices and even well-resourced institutions which can be burdened with increased cost, limitations in staffing, and patient preferences for more convenient options. Infusion site reactions and bruising are also fairly-common. Given these complications, alternative administration schedules that avoid weekend administration have been developed and evaluated. In the only randomized phase 2 trial to compare different azacitidine administration schedules in MDS, 151 patients were randomized in community-based centers to one of three weekend-less subcutaneous regimens: azacitidine $75 \mathrm{mg} / \mathrm{m}^{2}$ daily for 5 days, followed by 2 days without treatment, then $75 \mathrm{mg} / \mathrm{m} 2$ daily for 2 days (or $5-2-2$ ); azacitidine $75 \mathrm{mg} / \mathrm{m}^{2}$ daily for 5 days $(5-0)$; or azacitidine $50 \mathrm{mg} / \mathrm{m}^{2}$ daily for 5 days, followed by 2 days without treatment, then $50 \mathrm{mg} / \mathrm{m}^{2}$ daily for 5 days (or $5-2$ 5) [10]. There were no significant differences in the HI rates $(44 \%, 56 \%$, and $45 \%)$ or in rates of red blood cell (RBC) transfusion-independence $(50 \%, 64 \%$, and 55\%) between 5 and 2-2, 5-0, and 5-2-5 groups, respectively. Importantly, most studied patients (63\%) in the trial had lower-risk MDS as defined by the French-American-
British (FAB) classification, namely refractory anemia, refractory anemia with ringed sideroblasts, and chronic myelomonocytic leukemia with $<5 \%$ bone marrow blasts $[10,11]$. Since a minority of patients in this study had higher-risk MDS, there was no arm which used the standard 7-0 regimen, and there were no survival data in this study, these results cannot reliably be extrapolated to guide therapy in higher-risk MDS patients in whom the 7-0 regimen remains the standard of care [10].

In their recent study, Shapiro et al. conducted a systematic review of the available data comparing the different azacitidine administration schedules used for treatment of MDS, explicitly the 5-0,5-2-2, and 7-0 regimens [12]. Attempts at conducting a meta-analysis were unsuccessful given the paucity of randomized controlled studies (4 of 130 included studies, or 3\%) evaluating differences in administration schedules and the significant heterogeneity of the observational studies, but an ORR for each regimen was obtained via pooled analyses of the included studies. Two observational retrospective studies which compared the 5-0 with the $7-0$ schedule were also included, but in only one of two studies the study of patients had higher risk MDS and neither study showed a significant difference in OS $[13,14]$. The pooled proportion analyses of ORR were $44.4 \%$ with 95\% CI $(42.4 \%-45.1 \%)$ for the $7-0$ schedule, $41.2 \%$ with 95\% CI $(39.2 \%-41.9 \%)$ for the $5-0$ schedule, and $45.8 \%$ with $95 \%$ CI $(42.6 \%-46.4 \%)$ for the 5-2-2 schedule. Shapiro et al. suggest that schedules administering seven days of azacitidine treatment with or without a weekend break might have higher ORR than the other five-day schedules, but the fact that pooled results of alternative regimens cannot be directly compared limits this conclusion especially in patients with higher risk MDS. Shapiro et al. duly caution that their analysis was limited by several significant biases, the heterogeneity in design of the studies included (most of which were retrospective or uncontrolled) as well as their reporting of metrics (namely ORR and OS but not CR), which precluded direct comparisons between schedules [12].

Most studies that have used the 5-0 azacitidine regimen, including the only randomized prospective study comparing differing schedules by Lyons et al., were comprised of a clear majority of lower-risk MDS patients [Table 1] [10]. It is also worth noting that the observed effect on survival as demonstrated in the influential AZA-001 trial has not been replicated in real-world or population studies of higher-risk MDS patients. For example, a study of one of the largest reported cohort of patients with HMA-treated higher-risk MDS in the USA demonstrated that the median OS for patients treated with azacitidine was 16.4 months (95\% CI, 15.017.9 months) [15]. Another study using the Surveillance, Epidemiology, and End Results (SEER)-Medicare linked 
database showed that median OS with azacitidine among older patients with refractory anemia with excess blasts was 11 months [16]. One theory that has been proposed to explain the substantial difference in survival between real-life analyses and AZA-001 for patients with higherrisk MDS treated with azacitidine is the significant underuse of the 7-0 regimen in the community setting in favor of alternative administration schedules in the USA [17]. Indeed, one registry in the USA suggested that the 7-0 regimen is only used $15 \%$ of the time [18]. However, registry and population studies from Europe, in which patients often receive the approved 7-0 regimen, also reported significantly worse survivals than AZA-001 $[14,19,20]$. Therefore, it is likely that other factors aside from the administration schedule account for the substantially worse performance of azacitidine in the real-life setting in patients with higher-risk MDS [4, 19, 21-23].

On a mechanistic level, there are no well-established minimal effective doses for HMAs, including azacitidine [24]. The precise mechanism of action of azacitidine in MDS remains poorly defined despite extensive research and clinical use of the drug for almost 15 years now [25]. While, as its class name implies, epigenetic modification and hypomethylation are central to the prevailing theory regarding their mechanism of action, immunemediated and other effects might be in play [26]. Incorporation of azacitidine in RNA and DNA appear central to its activity, and with its short half-life combined with the need for the cells to be in the S-phase of the cell cycle for the drug to be incorporated into the DNA, it has been proposed that prolonged exposures to azacitidine via longer administrated schedules might be better pharmacodynamically in increasing the chance of targeting slow-cycling MDS cells [27]. Lower-dose, but prolonged schedules of azacitidine at $50 \mathrm{mg} / \mathrm{m}^{2}$ via the 5-2-5 schedule has been previously shown to effectively reverse promoter methylation [28]. Clinical metrics were also validated by the US Leukemia Intergroup Randomized Phase Study E1905 in which the 5-2-5 schedule resulted in a targeted $30 \%$ trilineage hematologic response rate, approximately double that seen in the AZA-001 study [27]. However, the survival associated with this regimen appeared worse than that of the 7-0 regimen in the AZA-001 trial, keeping in mind all the problems that arise in cross-trial comparisons. A phase I study of the oral formulation of azacitidine (CC-486) administered via the 7-0 schedule to a majority of lower-risk MDS patient has demonstrated an ORR of $73 \%$ and favorable side effect profile [29]. Fourteen and 21-day administration schedules of CC-486 have also demonstrated substantial ORR [30]. Perhaps avoiding the infusion requirement is the more convenient approach to deliver longer exposure to azacitidine, of course presuming a comparable or superior efficacy. A phase III multi-center randomized double-blind study is currently recruiting lower-risk MDS patients to be treated with the oral formulation using a prolonged administration schedule [31]. To further cloud the proposition that extended administration and thus extended exposure translates to better outcomes, a recent study has suggested that a shorter, three-day course of azacitidine for lower-risk MDS results in a significant ORR and CR rates of $49 \%$ and $36 \%$, respectively [32]. This grossly parallels the ORR and CR rates noted in prospective studies specifically evaluating 5-day regimens and the approved 7-day regimens, however there has been no direct comparison to the 7-0 regimen and patients with higher-risk MDS were not treated in this trial $[2-5,33-35]$.

\section{Conclusions}

In summary, inconsistent data with regards to the optimal use and schedules of azacitidine in both higher-risk and lower-risk MDS is leaving a lot of uncertainty regarding the best ways to administer this drug. When put in the context of clinical considerations and logistical difficulties, extended azacitidine administration regimens (e.g. 7-0) are often cumbersome. However, given the lack of convincing data otherwise, the approved 7-0 azacitidine schedule remains the only regimen associated with a survival advantage and is the recommended treatment for higher-risk MDS [1, 36]. While the 5-2-2 regimen is considered by many experts to be equivalent to the 7-0 regimen based on indirect comparison data [33, 34], there are no randomized prospective data comparing to $7-0$ regimen to support that notion. On the other hand, in lower risk MDS where the focus is on quality of life improvement and where no drug or regimen has prolonged survival, alternative (shorter or potentially longer) azacitidine regimens are generally appropriate. Ultimately, randomized clinical trials directly comparing azacitidine administration schedules in both lower risk and higher risk MDS patients are needed to guide the best practice in the community.

\section{Abbreviations}

AML: Acute myeloid leukemia; BSC: Best supportive care; CALGB: Cancer and leukemia group B; Cl: Confidence interval; CR: Complete response;

FAB: French-American-British; HI: Hematological improvement; HMA: Hypomethylating agent(s); IPSS: International prognostic scoring system; IWG: International Working Group; MDS: Myelodysplastic syndrome(s); ORR: Overall response rate; OS: Overall survival; PR: Partial response improvement; RBC: Red blood cell; SEER: Surveillance, epidemiology, and end results; USA: United States of America

\section{Acknowledgements}

Not applicable.

Funding

Not applicable. 


\section{Availability of data and materials}

Data sharing not applicable to this article as no datasets were generated or analyzed during this commentary.

\section{Authors' contributions}

RS drafted the article. Both authors wrote the manuscript and approved the final version.

\section{Ethics approval and consent to participate} Not applicable.

\section{Consent for publication}

Not applicable.

\section{Competing interests}

The authors declare that they have no competing interests.

\section{Publisher's Note}

Springer Nature remains neutral with regard to jurisdictional claims in published maps and institutional affiliations.

\section{Author details}

'Department of Internal Medicine, School of Medicine, Yale University, New Haven, CT, USA. ${ }^{2}$ Cancer Outcomes, Public Policy, and Effectiveness Research (COPPER) Center, Yale University, New Haven, CT, USA. ${ }^{3}$ Section of Hematology, Department of Internal Medicine, Yale University, 333 Cedar Street, PO Box 208028, New Haven, CT 06520-8028, USA.

Received: 18 October 2017 Accepted: 4 January 2018

Published online: 01 February 2018

\section{References}

1. Greenberg PL, Stone RM, Al-Kali A, et al. Myelodysplastic syndromes, version 2.2.107, NCCN clinical practice guidelines in oncology. J Natl Compr Cancer Netw. 2017:15(1):60-87.

2. Silverman LR, Holland JF, Weinberg RS, et al. Effects of treatment with 5 azacytidine on the in vivo and in vitro hematopoiesis in patients with myelodysplastic syndromes. Leukemia. 1993;7(Suppl 1):21-9.

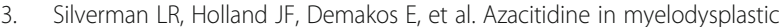
syndromes: CALGB studies 8421 and 8921. Ann Hematol. 1994;68:A12.

4. Fenaux P, Mufti GJ, Hellstrom-Lindberg E, et al. Efficacy of azacitidine compared with that of conventional care regimens in the treatment of higher-risk myelodysplastic syndromes: a randomised, open-label, phase III study. Lancet Oncol. 2009:10:223-32.

5. Silverman $L R$, Demakos EP, Peterson BL, et al. Randomized controlled trial of azacitidine in patients with the myelodysplastic syndrome: a study of the cancer and leukemia group B. J Clin Oncol. 2002;20:2429-40.

6. Kantarjian $\mathrm{H}$, Issa JP, Rosenfeld CS, et al. Decitabine improves patient outcomes in myelodysplastic syndromes: results of a phase III randomized study. Cancer. 2006;106:1794-803.

7. Cheson BD, Greenberg PL, Bennett JM, Lowenberg B, Wijermans PW, Nimer SD, Pinto A, Beran M, de Witte TM, Stone RM, Mittelman M, Sanz GF, Gore SD, Schiffer CA, Kantarjian H. Clinical application and proposal for modification of the international working group (IWG) response criteria in myelodysplasia. Blood. 2006;108(2):419-25.

8. Gore SD, Fenaux P, Santini $V$, et al. A multivariate analysis of the relationship between response and survival among patients with higher-risk myelodysplastic syndromes treated within azacitidine or conventional care regimens in the randomized AZA-001 trial. Haematologica. 2013;98(7):1067-72.

9. Lubbert M, Suciu S, Baila L, et al. Low-dose decitabine versus best supportive care in elderly patients with intermediate- or high-risk myelodysplastic syndrome (MDS) ineligible for intensive chemotherapy: final results of the randomized phase III study of the European Organisation for Research and Treatment of Cancer leukemia group and the German MDS study group. J Clin Oncol. 2011:29:1987-96.

10. Lyons RM, Cosgriff TM, Modi SS, et al. Hematologic response to three alternative dosing schedules of azacitidine in patients with myelodysplastic syndromes. J Clin Oncol. 2009;27:1850-6.

11. Bennett JM, Catovsky D, Daniel MT, et al. Proposals for the classification of the myelodysplastic syndromes. Br J Haematol. 1982;51:189-99.
12. Shapiro R, lansavichene A, Lazo-Langner A. Systematic review of azacitidine regimens in myelodysplastic syndrome and acute myeloid leukemia. BMC Hematol: In press; 2017.

13. Xicoy B, Jimenez MJ, Garcia O, et al. Results of treatment with azacitidine in patients aged $\geq 75$ years included in the Spanish registry of Myelodysplastic syndromes. Leuk Lymphoma. 2014;55(6):1300-3.

14. Garcia-Delgado R, de Miguel D, Bailen A, et al. Effectiveness and safety of different azacitidine dosage regimens in patients with myelodysplastic syndromes or acute myeloid leukemia. Leuk Res. 2013;38(7):744-50.

15. Zeidan AM, Sekeres MA, Garcia-Manero G, et al. Comparison of risk stratification tools in predicting outcomes of patients with higher-risk myelodysplastic syndromes treated with azanucleosides. Leukemia. 2016;30:649-57.

16. Zeidan AM, Davidoff AJ, Long JB, et al. Comparative clinical effectiveness of azacitidine versus decitabine in older patients with myelodysplastic syndromes. Br J Haematol. 2016;175(5):829-40.

17. Zeidan AM. Hypomethylating agents in myelodysplastic syndromes and population-level outcomes: a changing landscape or a small dent? Leuk lymphoma. Sept. 2017;27:1-3

18. Grinblatt DL, Sekeres MA, Komrokji RS, et al. Patients with myelodysplastic syndromes treated with azacitidine in clinical practice: the AVIDA registry. Leuk Lymphoma. 2015;56(4):887-96.

19. Bernal T, Martinez-Camblor P, Sanchez-Garcia J, et al. Effectiveness of azacitidine in unselected high-risk myelodysplastic syndromes: results from the Spanish registry. Leukemia. 2015;29:1875-81.

20. van der Helm LH, Alhan C, Wijermans PW, et al. Platelet doubling after the first azacitidine cycle is a promising predictor for response in myelodysplastic syndromes (MDS), chronic myelomonocytic leukaemia (CMML) and acute myeloid leukaemia (AML) patients in the Dutch azacitidine compassionate named patient programme. Br J Haematol. 2001; 155(5):599-606.

21. Sekeres MA, Othus M, List AF, et al. Randomized phase II study of azacitidine alone or in combination with lenalidomide or with vorinostat in higher-risk myelodysplastic syndromes and chronic myelomonocytic leukemia: north American intergroup study SWOG S1117. J Clin Oncol. 2017:35:2745-53.

22. Zeidan AM, Stahl M, Sekeres MA, et al. A call for action: increasing enrollment of untreated patients with higher-risk myelodysplastic syndromes in first-line clinical trials. Cancer. 2017;123:3662-72.

23. Zeidan AM, Wang R, Gross CP, et al. Modest improvement in survival of patients with refractory anemia with excess blasts in the hypomethylating agents era in the United States. Leuk Lymphoma. 2017:58(4):982-5.

24. Zeidan AM, Linhares Y, Gore SD. Current therapy of myelodysplastic syndromes. Blood Rev. 2013;27(5):243-59.

25. Abou Zahr A, Saad Aldin E, Barbarotta L, et al. The clinical use of DNA methyltransferase inhibitors in myelodysplastic syndromes. Expert Rev Anticancer Ther. 2015:15(9):1019-36.

26. Abou Zahr A, Bernabe Ramirez C, Wozney J, et al. New insights into the pathogenesis of MDS and the rational therapeutic opportunities. Expert Rev Hematol. 2016;9(4):377-88

27. Prebet $T$, Sun Z, Figueroa ME, et al. Prolonged administration of azacitidine with or without entinostat for myelodysplastic syndrome and acute myeloid leukemia with myelodysplasia-related changes: results of the US Leukemia Intergroup trial E1905. J Clin Oncol. 2014;32(12):1242-8.

28. Gore SD, Baylin S, Sugar E, et al. Combined DNA methyltransferase and histone deacetylase inhibition in the treatment of myeloid neoplasms. Cancer Res. 2006:66:6361-9.

29. Garcia-Manero G, Gore SD, Cogle C, et al. Phase 1 study of oral azacitidine in myelodysplastic syndromes, chronic myelomonocytic leukemia, and acute myeloid leukemia. J Clin Oncol. 2011;29(18):2521-7.

30. Garcia-Manero G, Gore SD, Kambhampati S, et al. Efficacy and safety of extended dosing scheduled of CC-486 (oral azacitidine) in patients with lower-risk myelodysplastic syndromes. Leukemia. 2016;30(4):889-96.

31. A Phase 3, Multicenter, Randomized, Double-blind Study to Compare the Efficacy and Safety of Oral Azacitidine Plus Best Supportive Care Versus Placebo Plus Best Supportive Care in Subjects With Red Blood Cell Transfusion-dependent Anemia and Thrombocytopenia Due to IPSS Lowerrisk Myelodysplastic Syndromes. 2012. Retrieved from https://clinicaltrials. gov/ct2/show/NCT01566695?term=NCT01566695\&rank=1 (Identification No. NCT01566695).

32. Jabbour E, Short NJ, Montalban-Bravo G, et al. Randomized phase 2 study of low-dose decitabine vs low dose azacitidine in lower-risk MDS and MDS/ MPN. Blood. 2017;130(13):1514-22. 
33. Breccia M, Loglisci G, Salaroli A, et al. 5-Azacitidine efficacy and safety in patients aged $>65$ years with myelodysplastic syndromes outside clinical trials. Leuk Lymphoma. 2012;53(8):1558-60.

34. Breccia M, Loglisci G, Cannella L, et al. Application of French prognostic score to patients with InternationalPrognostic scoring system intermediate-2 or high risk myelodysplasticsyndromes treated with 5azacitidine is able to predict overall survival and rate of response. Leuk Lymphoma. 2012;53(5):985-6.

35. Fili C, Malagola M, Follo MY, et al. Prospective phase II study on 5-days Azacitidine for treatment of symptomatic and/or erythropoietin unresponsive patients with low/INT-1-risk Myelodysplastic syndromes. Clin Cancer Res. 2013;19(12):3297-308

36. Fenaux P, Bowen D, Gattermann N, et al. Practical use of azacitidine in higher-risk myelodysplastic syndromes: an expert panel opinion. Leuk Res. 2010;34:1410-6.

37. Martin MG, Walgren RA, Procknow E, et al. A phase II study of 5-day intravenous azacitidine in patients with myelodysplastic syndromes. Am J Hematol. 2009:84:560-4.

38. Tobiasson M, Dybedahl I, Holm MS, et al. Limited clinical efficacy of azacitidine in transfusion-dependent, growth factor-resistant, low- and Int-1risk MDS: results from the nordic NMDSG08A phase II trial. Blood Cancer J. 2014:4:1-7.

\section{Submit your next manuscript to BioMed Central} and we will help you at every step:

- We accept pre-submission inquiries

- Our selector tool helps you to find the most relevant journal

- We provide round the clock customer support

- Convenient online submission

- Thorough peer review

- Inclusion in PubMed and all major indexing services

- Maximum visibility for your research

Submit your manuscript at www.biomedcentral.com/submit 